Trauma Berufskrankh 2009 • 11[Suppl 1]:22-23 DOI 10.1007/s10039-008-1451-4

๑) Springer Medizin Verlag 2009

\author{
V. Bühren \\ BG-Unfallklinik, Murnau
}

\title{
Traumanetzwerk vs. BG-Verfahren
}

werden Statistiken und Literatur zur Epidemiologie und Versorgungsstruktur des schweren Traumas für den deutschen und internationalen Schriftraum. Wesentliche Rückschlüsse für die deutschen Verhältnisse bietet mit hoher Datenqualität das Polytraumaregister der DGU [6].

Für Deutschland wird eine 3-stufige Gliederung der Schwerverletztenversorgung mit einer flächendeckenden Basisstruktur und darauf aufbauenden regionalen und überregionalen Traumazentren vorgeschlagen. Die personellen und logistischen Anforderungen für diese so definierte Maximal-, Schwerpunkt- und Flächenversorgung wurden aktualisiert zum ursprünglichen Grundlagenpapier von 1997 definiert [4].

Die rechtliche Grundlage für das Weisungsrecht zur Definition von Zulassungsanforderungen in den BG-lichen Heilverfahren liefert der $\$_{34}$ des Sozialgesetzbuchs (SGB) VII für die Gesetzliche Unfallversicherung. Für den stationären Bereich sind das Durchgangsarzt- (D-Arzt-) und das Verletzungsartenverfahren (VAV) mit personellen, räumlichen und strukturellen Voraussetzungen niedergelegt [3]. Persönliche Qualifikationen sind für beide HV der Facharzt Chirurgie oder Orthopädie/Unfallchirurgie sowie Zusatzbezeichnungen wie die Schwerpunktbezeichnung Unfallchirurgie oder die Bezeichnung Spezielle Weiterbildung Unfallchirurgie. Für das VAV wird weiterhin eine mindestens 2-jährige Weiterbildungsbefugnis in der speziellen Unfallchirurgie gefordert.

\section{Kalkulation}

Die Leistungsfähigkeit unfallchirurgischer Abteilungen lässt sich über die Faktoren
Betten- und Operationskapazität, das operative Spektrum und die Fallzahlen mit guter Reproduzierbarkeit beschreiben [1]. Objektivierbare Zahlen werden über die Operationsdokumentation, Fallzahlen und die Jahresmeldungen an die Landsverbände im VAV geliefert. Hieraus lässt sich eine 1-, 2- oder Mehrzügigkeit einer unfallchirurgischen Klinik ableiten.

Ein ganztägig benutzter Operationssaal (OP) im Vollbetrieb in der Unfallchirurgie hat eine Kapazität von etwa 1000 Eingriffen/Jahr, gezählt nach Narkosen. Entsprechend der üblichen Liegedauer von 7 Tagen werden bei einer 80\%igen Auslastung für den Betrieb etwa 25 Betten benötigt. Erfahrungsgemäß sind 1/5 der operierten Fälle in der Unfallchirurgie bezüglich des Schweregrades analog des Schwerverletztenartenkatalogs des VAV einzuordnen. Entsprechend sind bei einer relativen Konstanz mit 20\% Anteil BG-lich Versicherter 50 Fälle des VAV pro Jahr zu erwarten.

Eine 1-Zügigkeit entspricht somit 25 Betten, einem Vollzeit-OP, 1000 operativen Fällen und einer Übungshaltung von 250 relevanten Verletzungen pro Jahr, was 1 Fall täglich entspricht. Für die Schwerpunktversorgung ist eine doppelte Kapazität mit 2 Sälen und mindestens 50 Betten erforderlich. Die Aufgaben der Maximalversorgung sind nur in 3- oder mehrzügigen Abteilungen zu bewältigen.

\section{Synergien}

Für Industrieländer mit hohem Versorgungsstandard kann der Bedarf für maximal versorgende Traumazentren mit et-

Übernahme aus Trauma und Berufskrankheit 2008 $\cdot 10$ [Suppl 1]: 93-94 
wa 1/1.000.00o Einwohner definiert werden. In Deutschland sind derzeit mehr als 500 Kliniken zum VAV zugelassen. Aus den vorgenannten Kalkulationen und den Vorgaben des Weißbuchs lässt sich eine Dreigliedrigkeit der Schwerverletztenversorgung unter den Gesichtspunkten des Bedarfs und der Qualität formulieren.

Notwendig sind etwa 60 maximal versorgende Zentren mit jeweils mehr als 70 Betten und hoher Operationskapazität mit mindestens 3 Sälen parallel bei einem Durchsatz von 3000 operativen Fällen/ Jahr. Schwerpunkthäuser werden mit einer Zahl von 200-30o benötigt. Sie sollten zum VAV zugelassen sein und annähernd 100 Fälle des VAV pro Jahr behandeln. Diese Häuser werden in der Regel eine Weiterbildungsbefugnis von mindestens 2 Jahren von den zuständigen Landesärztekammern erhalten. Die flächendeckende Versorgung kann über 1zügige Abteilungen erfolgen, die in jedem Fall über eine Durchgangsarztzulassung verfügen sollten.

\section{Ausblick}

Die Initiative der Deutschen Gesellschaft für Unfallchirurgie zum Aufbau eines 3stufigen Traumanetzwerks in Deutschland kann durch die seit langem etablierten Heilverfahren der Deutschen Gesetzlichen Unfallversicherung (DGUV) wirksam unterstützt werden. Die HV bieten eine gesetzliche Verankerung für die geforderten Ausstattungsstandards und Personalkompetenzen. Eine deckungsgleiche Ausprägung der Zulassung zum VAV einerseits und der Zertifizierung als regionales oder überregionales Traumazentrum ist anzustreben.

Qualitätssichernde Aspekte des TN wie die Teilnahme am Traumaregister sind auch als Verpflichtung zur Leistungsabbildung im VAV denkbar. Eine verbesserte nachvollziehbare Leistungserfassung kann im Sinne von Mindestmengen die Übungshaltung sicherstellen und eine am Bedarf adaptierte Verteilung der Traumastützpunkte unterstützen. Die Auslegung des TN als lernendes System einerseits und die dynamische Weiterentwicklung der HV unter einer künftigen zentralen einheitlichen Deutschen Gesetzlichen Unfallversicherung anderer-
Internetlinks

- DGU: http://www.dgu-online.de

- DGUV: http://www.dguv.de

- LVBG: http://www.lvbg.de

- Traumanetzwerk de DGU: http://www. dgu-traumanetzwerk.de

seits bieten auch nach Zusammenschluss von Orthopädie und Unfallchirurgie erhebliche Chancen zur Sicherung einer nach internationalen Standards auf hohem Niveau strukturierten unfallchirurgischen Versorgung in Deutschland.

\section{Korrespondenzadresse}

\section{Prof. Dr. V. Bühren}

BG-Unfallklinik Murnau,

Prof. Küntscher-Straße 8, 82418 Murnau

buehren@bgu-murnau.de

Interessenkonflikt. Der korrespondierende Autor gibt an, dass kein Interessenkonflikt besteht.

\section{Literatur}

1. Bühren V (2007) Arithmetik der Traumaversorgung. Trauma Berufskrankh [Suppl] 1: 39-42

2. Deutsche Gesellschaft für Unfallchirurgie (2006) Weißbuch Schwerverletzten-Versorgung. Deutsche Gesellschaft für Unfallchirurgie, Berlin, http:// www.dgu-online.de/pdf/unfallchirurgie/weissbuch/weissbuch.pdf

3. Deutsche Gesetzliche Unfallversicherung (2005) Anforderungen in den Heilverfahren. Deutsche Gesetzliche Unfallversicherung, Berlin, http:// www.lvbg.de/lv/pages/aufgabe/m_reha/verletz/ index.html

4. Haas NP (1997) Empfehlungen zur Struktur, Organisation und Ausstattung der präklinischen und klinischen Patientenversorgung an Unfallchirurgischen Abteilungen in Krankenhäusern der Bundesrepublik Deutschland. Unfallchirurgie 100: 2-7

5. Kühne CA, Ruchholtz S, Buschmann C et al. (2006) Initiative Traumanetzwerk DGU: Polytraumaversorgung in Deutschland - eine Standardbestimmung. Unfallchirurgie 109: 357-366

6. Ruchholtz S, AG Polytrauma (2000) Das Traumaregister der Deutschen Gesellschaft für Unfallchirurgie als Grundlage des interklinischen Qualitätsmanagements in der Schwerverletztenversorgung. Unfallchirurgie 103: 30-37
Trauma Berufskrankh 2009 11

[Suppl 1]:22-23

DOI 10.1007/s10039-008-1451-4

(c) Springer Medizin Verlag 2009

\section{Bühren}

\section{Traumanetzwerk}

\section{vs. BG-Verfahren}

\section{Zusammenfassung}

Auf der Grundlage des Weißbuchs zur unfallchirurgischen Versorgung von Schwerverletzten soll auf Initiative der Deutschen Gesellschaft für Unfallchirurgie ein Traumanetzwerk installiert werden. Seit Jahrzehnten bestehen im Rahmen des Versorgungsauftrags der Gesetzlichen Unfallversicherung definierte Ausstattungs- und Personalanforderungen an zugelassene unfallchirurgische Abteilungen. Über objektivierbare Leistungs- und Ausstattungsparameter lassen sich zweckmäßige Abteilungsgrößen für die flächendeckende Versorgung sowie die übergreifenden regionalen und überregionalen Traumazentren in einem 3-stufigen System definieren. Beide Systeme bieten prinzipielle Gestaltungsmöglichkeiten für eine optimale qualitätsorientierte und bedarfsgerechte Versorgungssituation.

\section{Schlüsselwörter}

Versorgungsauftrag · Traumanetzwerk .

Traumazentrum · Gesetzliche

Unfallversicherung · Weißbuch

\section{Trauma network versus legal accident insurance system}

\section{Abstract}

Based on the so-called white paper on the structure for treating severely injured patients, a trauma network should be installed on the initiative of the German Society for Trauma Surgery. For decades, equipment and staff requirements for authorized surgical departments have been defined by the legal accident insurance industry. Regarding parameters for performance and equipment, department sizes have been defined for regional and supraregional trauma centers in a three-stage system. Both systems offer principle design options for an optimal qualityoriented supply situation adapted to specific needs.

\section{Keywords}

Duty of medical care - Trauma network . Trauma center - Legal accident insurance . White paper 